\title{
Tangence
}

\section{Aventures des imaginaires de la dissidence et de la marginalité de Jean-Jacques Rousseau à Jean-Paul Marat}

Jean M. Goulemot

Numéro 57, mai 1998

Littérateurs atypiques et penseurs irréguliers

URI : https://id.erudit.org/iderudit/025965ar

DOI : https://doi.org/10.7202/025965ar

Aller au sommaire du numéro

Éditeur(s)

Tangence

ISSN

0226-9554 (imprimé)

1710-0305 (numérique)

Découvrir la revue

Citer cet article

Goulemot, J. M. (1998). Aventures des imaginaires de la dissidence et de la marginalité de Jean-Jacques Rousseau à Jean-Paul Marat. Tangence, (57), 12-22. https://doi.org/10.7202/025965ar d'utilisation que vous pouvez consulter en ligne. 


\title{
Aventures des imaginaires de la dissidence et de la marginalité de Jean-Jacques Rousseau à Jean-Paul Marat
}

\author{
Jean M. Goulemot, \\ Institut universitaire de France, Université de Tours
}

Pas plus que le navigateur avisé ne s'embarque sans biscuit, sans whisky et sans eau fraîche, le chercheur en quête de marginalité littéraire ne peut faire l'économie d'une réflexion sur son objet. Car des marges et des marginaux, il va en trouver à foison. Non des bannis sociaux, Vautrin et Vidocq des temps nouveaux, tire-laine ou picaros des temps plus anciens, que la littérature mettrait en scène et se choisirait comme autant de héros ${ }^{1}$, mais de ces littérateurs en rupture d'orthodoxie, hors normes esthétiques et littéraires. Et ce dès le Moyen-Âge: goliards et autres clercs errants $^{2}$, plus tard libertins érudits ${ }^{3}$ ou non, habitués forts en gueule et pentus du gosier du Cabaret de la Pomme de Pin, lestes en gestes et en écrits, rétifs à l'ordre esthétique nouveau des débuts de l'âge classique ${ }^{4}$. Ils sont foison, sans compter même pornographes et autres écrivains gaillards ${ }^{5}$. À la fin du $\mathrm{xvII}^{\mathrm{e}}$ siècle, le Refuge propose quelques belles figures d'excentriques: comme Nicolas Gueudeville ${ }^{6}$, moine apostat, protestant hétérodoxe, continuateur des dialogues de La Hontan, rédacteur

1 Ainsi que clans les romans de gueuserie, dont Roger Chartier a mené l'analyse. Cf. Figures de la gueuserie, textes présentés par Roger Chartier, Paris, Montalba, coll. "Bibliothèque bleue", 1982.

2 Marie-Claude de Crécy, Vocabulaire de la littérature du Moyen-Âge, Paris, Minerve, 1997.

3 René Pintard, Le libertinage érudit: dans la première moitiê du xvIr siècle, Genève, Slatkine, 1981 (1943).

4 On verra Antoine Adam, Histoire de la littérature française au xvir siècle, Paris, Albin Michel, coll. "Bibliothèque de l'Évolution de l'Humanité", 1997 (rééd.), 4 vol., mais aussi Georges Mongrédien, La vie littéraire au Xvfr siècle, Paris, Taillandier, 1952.

5 Pornographe comme Paul Alexis, dit Pierre Comeille de Blessebois (16461697), auteur de Le rut ou la pudeur éteinte.

6 Sur Nicolas Gueudeville, on verra Aubrey Rosenberg, Nicolas Gueudeville and bis Work, La Haye/Boston, London, "International Archives of the History of Icleas", 1982. 
du sulfureux Esprit des Cours $^{7}$ et d'autres brûlots antimonarchiques $^{8}$, ou encore le pasteur Pierre Jurieu, ennemi juré de Pierre Bayle, dont le rapport à la norme était, comme l'on sait, problématique (qu'on examine ses positions sur l'obscénité en ce siècle policé 9 ), tenant lui d'une orthodoxie inquisitoriale et farouche, visionnaire à ses heures et passablement extravagant ${ }^{10}$. Le milieu protestant fourmille de ces fous de Dieu, hallucinés et marginaux comme Annette Bourignon ${ }^{11}$ qui affirmait à ses disciples qu'avant la Faute Adam possédait, en guise d'attributs virils, un appendice nasal étrangement situé et propre à capter les divines senteurs de l'Eden.

Si nous examinions la seconde moitié du xvIII ${ }^{\mathrm{e}}$ siècle, preuve que le rationalisme claironné ne fait pas pour autant de la raison la chose du monde la mieux partagée, la moisson serait plus abondante encore. À commencer par Jean-Jacques Rousseau que nombre de ses contemporains, moins indulgents que la postérité, jugeaient comme un original, un excentrique ou un extravagant. N'avait-il pas refusé d'être présenté au roi, n'avait-il pas abandonné bas de soie, montre et habit de cour, ne vivait-il pas loin des hommes et des villes, revêtu de son habit d'Arménien, n'affirmait-il pas enfin le contraire de ce que pensait la majorité des honnêtes gens et des hommes d'esprit? Il fallut le succès de $L a$ nouvelle Héloïse (une concession aux goûts d'un siècle amateur de romans, dont il lui fallut s'expliquer) pour que l'homme des marges, de la rupture fût accepté avec ses lubies et ses paradoxes, que l'on avait sans doute pris à l'origine comme autant de coquetteries littéraires. Pour qui s'en tiendrait à une définition assez souple des "fous littéraires", Jean-Jacques pourrait passer pour un "enfant du limon". Tout y est: le talent, la reconnaissance générale, l'acceptation sérieuse et parfois même révéren-

7 Ce périodique a pour titre exact L'esprit des cours de l'Europe, où l'on voit tout ce qui s'y passe de plus important touchant la politique, et en général ce quil $y$ a de plus remarquable dans les nouvelles.

8 J'ai analysé la portée idéologique de tels textes dans Jean Marie Goulemot, Le règne de l'Histoire: discours bistoriques et révolutions, Paris, Albin Michel,1996, p. 281 et ss.

9 Voir Jean Christophe Abramovici, Le livre interdit, Paris, Payot, 1996.

10) En particulier quand il sombra dans le prophétisme et dénonça en Louis XIV l'incarnation de l'Ante-Christ.

11 Annette Bourignon (1616-1680), "rêveuse mystique", dont Pierre Bayle et Voltaire se sont moqués. Elle a, par la plume de son disciple Pierre Poiret, laissé une cuvre théologico-mystique en 19 volumes. 
14

tielle de l'œuvre en plus. D'abord l'esprit de système, la Révélation d'une explication globale du monde, du destin de l'homme et de la société, le comportement marginal, la lecture exagérément interprétative, inquiète et passionnée, des signes sociaux comme le prouvent les hallucinations auditives dont témoignent Les confessions et le dédoublement inquisitorial des Dialogues. Ce qui dans l'œuvre et le comportement de Rousseau va frapper, non ses contemporains, primitifs ou non de la culture, qui n'y regardent pas de si près, mais ses pairs, c'est ce rôle de juge lointain et sévère que s'octroie Jean-Jacques, - Diderot en nourrira une haine pour son ancien ami que la mort elle-même ne parviendra pas à éteindre ${ }^{12}$ - et la légitimation, qu'à partir de lui, il constitue pour son écriture et sa philosophie. La marge, le refus du monde, l'excentricité du vêtement et du mode de vie font de Rousseau aux yeux du plus grand nombre un objet de curiosité publique dont il s'étonne et qui ne cesse de l'inquiéter. On défile rue Plâtrière, quand il prend le frais ou la pose à sa fenêtre, en compagnie de Thérèse ${ }^{13}$. L'aristocratie se passionne pour l'original penseur: elle l'accueille, recueille la moindre de ses reliques, achète à grands frais ses herbiers, ses manuscrits et ses travaux de copiste. L'accord est unanime avec des formes propres. Pour Rousseau la solitude, la marge, l'écart sont le prix à payer pour accéder à la vertu, à l'authenticité (la coïncidence du dire et du faire). Ne pas en être, ne pas faire partie du monde patenté des Lettres, à la plume servile, devient la possibilité de la vertu, condition nécessaire et suffisante pour dire vrai. L'idéologie rousseauiste recueille avec une évidente complaisance le modèle érémitique. Le vicaire savoyard est plus près des grottes sombres et moussues que des lumineux sommets alpins.

Et pourtant qui oserait faire de Rousseau un "fou littéraire"? Le succès rencontré par sa philosophie, le culte collectif dont il fut l'objet, la prise en compte historique de son influence, le champ qu'elle offrait au commentaire ou à la polémique, en dehors

12 On relira le jugement que porte sur Rousseau, déjà mort, Denis Diderot dans La vie de Sénèque. De fidèles rousseauistes, dont Anacharsis Cloots, vengèrent leur idole en brûlant un exemplaire dudit livre sur la tombe même de JeanJacques. On connaît la défense de Jean-Jacques entreprise par Robespierre, plaçant Rousseau contre le clan encyclopédiste jugé comme celui des nantis.

13 Voir le témoignage de Jacques-Louis Ménétra: Journal de ma vie: JacquesLouis Ménétra, compagnon vitrier au XnII siècle, présenté par Daniel Roche, Paris, Montalba, 1982. 
même de toute valorisation littéraire ou philosophique, rendent une telle qualification non seulement incongrue, mais impossible. Qu'il ait été considéré comme "un original", et parfois comme bien plus que cela par nombre de ses contemporains, ne change rien à l'affaire. Le succès est là qui occulte les réserves et les jugements sévèrement critiques des uns et des autres: de d'Holbach à Grimm en passant par Diderot, et sans oublier tous ceux qui ont clisserté de la pathologie de sa personnalité et de son œuvre.

À bien des égards donc il faudra admettre que ce sont, pour une large part, ses lecteurs et le rousseauisme qui ont sauvé Rousseau de la marginalité, au sens où l'on emploie le terme pour désigner un écrivain mineur et excentrique. On distinguera dans cette lecture et cette appropiation salvatrices divers niveaux, qu'on ne peut sans risque de fausser les perspectives, confondre totalement. Il existe, soyons-en convaincus, passablement hétérogènes, un imaginaire rousseauiste de ses lecteurs et plus largement de l'opinion, et une figure de Rousseau à usage spécifiquement littéraire. L'un et l'autre se retrouvent dans l'invocation du cher Jean-Jacques qui unit en apparence les visiteurs de Montmorency et les écrivains en quête de gloire ou d'émotions lyriques: de Bernardin de Saint-Pierre à Chateaubriand.

Ce qui d'entrée regarde ici notre analyse, c'est ce par quoi la vie écrite de Jean-Jacques accédant à l'écriture, ainsi que la constitue lui-même Jean-Jacques Rousseau, va fonder un imaginaire nouveau de l'écrivain et de l'écriture ${ }^{14}$. Le récit des Confessions marque à jamais le tempo, indique la pose, donne les indications de mise en scène de l'écrivain moderne ${ }^{15}$. Je n'insisterai pas ici sur la biographie nécessaire qui sera celle de l'écrivain postrousseauiste: la séparation d'avec la mère (le fait peut connaître de multiples variations, comme le prouve le cas Baudelaire), le goût prématuré des livres et de la lecture, la conquête obsessionnelle de l'écriture... Plus essentielle me semble encore, dans la perspective de réflexion que nous avons tenté de définir, l'importance que le système rousseauiste donne, pour se légitimer, à la différence, à la marginalité. L'écrivain-philosophe se définit contre. Contre d'autres écrivains, reconnus, domestiqués, intégrés

14 Jean Maric Goulemot, "Ine autobiographie d'écrivain", dans Littérature, févricr $1979, \mathrm{n}^{\circ} 33$.

15 L'expression de "mise en scène "est empruntéc à Daniel Oster, L'individu littéraire, Paris, Presses universitaires de France, coll. "Écriture", 1997. 
à la vie littéraire, faisant partie, comme l'on dirait aujourd'hui du système. Rousseau n'a pas de mots trop durs pour dénoncer masques et hypocrisies mensongères de ceux qu'il appelle des folliculaires ${ }^{16}$ "[...] chacun se met sans cesse en contradiction avec lui-même sans qu'on s'avise de le trouver mauvais. On a des principes pour la conversation et d'autres pour la pratique; leur opposition ne scandalise personne. On n'exige pas d'un Auteur, surtout d'un moraliste qu'il parle comme ses livres, ni qu'il agisse comme il parle. Ses écrits, ses discours, sa conduite sont trois choses différentes qu'il n'est pas obligé de concilier..." ${ }^{17}$ Le procès ne porte pas sur la fausseté du discours tenu par les littérateurs. Un tel procès viendra plus tard quand la dialectique de Jean-Jacques se sera affinée. La contradiction ici est seule en cause. "Faites ce que je dis et non ce que je fais." La première dénonciation du monde des Lettres par Jean-Jacques est celle d'un moraliste et non d'un philosophe en quête de légitimité. La vérité n'entre pas en ligne de compte dans le jugement porté sur les folliculaires et les écrivains mondains. Rousseau veut faire carrière. D'où sa méthode de notation musicale présentée au jugement de Réaumur, d'où aussi la lecture du Mercure et le concours académique dijonnais, quand bien même tout cela est raconté dans Les confessions à la façon d'une illumination mystique ${ }^{18}$. C'est sans aucun doute aussi la raison d'être du Discours sur les Sciences et les Arts, sa forte orientation morale et les ambiguités qui demeurent quant à la fermeté alors de la position rousseauiste: pour ou contre le progrès ${ }^{19}$. La polémique qui suit le discours, l'habileté courtoise qu'y déploie Jean-Jacques prouvent qu'il n'a pas abandonné ses projets de réussite littéraire et sociale. Tout laisse penser qu'il croit alors qu'on peut être un écrivain philosophe vertueux et vivre dans le monde, un écrivain dont le dire et le faire ne seraient pas contradictoires. C'est peu à peu, soyons-en sûr, que le doute s'insinue et que la lucidité s'impose. Rousseau va rompre avec ce destin au moment même où la carrière s'ouvre à lui. Le 19 octobre 1752, il quitte Fontainebleau

16 Cf. Jean Marie Goulemot, "Rousseau et les figures de l'intellectuel", dans Saggi e Ricercbe di Letteratura francese, vol. XXVIII, Rome, 1989.

17 Comme le dit Jean-jacques Rousscau dans le Discours sur les sciences et les arts.

18 Voir Les confessions, livre VIII.

19 C'est parfois ce qu'on prétend. Rousseau aurait hésité et Diderot lui aurait montré ce que la thèse hostile au progrès pouvait avoir de novateur. 
sans se rendre à l'audience royale où il était convoqué. C'est la célèbre réforme si souvent commentée. Rousseau choisit la campagne contre les salons, abandonne montre et bas de soie, se fait ermite avec Thérèse. Cette fracture va aller s'accentuant et il est inutile ici d'en suivre l'histoire. Rousseau creuse l'écart avec le monde littéraire, les institutions, se brouille avec ses amis, opte pour la solitude accusatrice tout en tentant un retour condamné à l'échec avec Genève où il se rend en 1754. En 1756 il s'installe à l'Ermitage, puis en 1757 à Montmorency. À la suite de la publication de la Lettre sur les spectacles, la rupture est consommée avec Diderot, Grimm et d'Holbach. C'est à partir des lettres autobiographiques à Malesherbes de 1762 que Rousseau, me semble-t-il, va faire de sa marginalité et des persécutions dont il est victime une arme et une légitimation. En même temps ou presque que le Parlement le décrète de prise de corps, que les autorités de Genève (Le Petit-Conseil) font mettre Le contrat social et L'Émile sous scellés. Son œuvre dès lors (La profession de foi du vicaire savoyard, La lettre à Cbristophe de Beaumont, Les lettres écrites de la Montagne, Les confessions et les Dialogues) semble obéir à une nouvelle nécessité : montrer que la vie légitime l'œuvre et lui donne sens et valeur. On connaît la démarche: l'exil social suscite la colère, le rejet des gens en place, mais il représente un choix vertueux et un moyen d'accéder à la vérité. La marginalité jugée négativement par ceux qui détiennent et gèrent l'institution littéraire, objet de curiosité pour l'opinion, va devenir, parce que librement choisie, le moyen nécessaire et suffisant de dire vrai. Le mouvement est infiniment complexe: Rousseau dit vrai parce qu'il a choisi contre un monde trompeur et corrompu la solitude vertueuse, et c'est parce qu'il dit vrai que le monde le voue à la marginalité. Le reste relève des apparences ou des signes ${ }^{20}$. Le célèbre habit d'arménien que revêt Rousseau a pour fonction de visualiser. Un texte comme Rousseau juge de Jean-Jacques met en scène, d'une façon hallucinée, les preuves de la vertu de l'écrivain, dénoncé ailleurs comme un fou insensé ou un hypocrite.

Sans pousser plus avant une telle analyse de la légitimation rousseauiste avec ce qu'elle implique de masque, de rôle, de mise en scène et de discours pédagogiques et justificatifs, on peut

20) On veut dire par là que Rousseau met en scène les signes de sá différence: elle sera vestimentaire, amoureuse, géographique, comme pour micux signifier qu'elle est morale et philosophique. 
en retenir un schéma: la marginalité fonde le droit non seulement à l'écriture mais à la verité, et il n'est, au fond, d'écrivain véritable que par opposition aux écrivains d'institution, que rejeté, rebelle, exclu. La fin du siècle sera habitée d'une foule innombrable d'exclus. Robert Darnton a montré comment se constitue à partir des années 70 une plèbe littéraire, exclue du marché du livre, vivant d'expédients et remâchant sa rancœur, rebelle, sûre d'être marquée d'un signe et d'être investie d'une mission ${ }^{21}$. Ces affamés, et pas seulement de gloire, rejetés et méconnus, obligés de se livrer à de viles besognes, conscients de leur déchéance, dénonçant l'institution littéraire, et parfois le monde politique, les accusant d'être responsables de leur avilissement, représentent pour l'historien de la littérature un objet fascinant parce que soumis à toutes les dérives, capable de tous les errements et de tous les recours. Là pullulent les marginaux et les excentriques, auteurs méconnus de systèmes esthétiques ou philosophiques aberrants, prêts à tous les plagiats et toutes les vilenies, passant de l'inspiration prophétique à la canaillerie littéraire ou sociale ${ }^{22}$. On peut y chercher des figures, souvent hautes en couleur de la marginalité littéraire, préfiguration des bohèmes qu'exaltera le $\mathrm{XIX}^{\mathrm{e}}$ siècle, mais aussi des excentriques, jouant à être, par goût des masques et nécessité de gagner le pain quotidien, maîtreschanteurs, agents secrets d'un pouvoir qu'ils détestent et accusent de tous les maux dont ils souffrent, plagiaires, maquereaux même. On pourrait ici s'arrêter sur la carrière de Pidansat de Mairobert, sur celle de Théveneau de Morande, ou mieux encore sur celle de Mercier de Compiègne, pornographe appliqué, prêt à chanter le vice, la vertu ou la république pour nourrir sa nombreuse progéniture ${ }^{23}$. Sans être des "fous littéraires", car ils n'ont

21 Robert Darnton, Bobème littéraire et Révolution: le monde des livres au xvmt siècle, Paris, Gallimard et Seuil, 1983; La fin des Lumières, Le Mesmérisme et la Révolution, Paris, Perrin, 1984; Edition et sédition: l'univers de la littérature clandestine au xvIf siècle, Paris, Gallimard, 1991; Gens de lettres et gens du livre, Paris, Odile Jacob, 1992.

22 Parmi les cas étudiés par Robert Darnton il est évident que le chantage voisine avec le pamphlet politique, la pornographie avec le putanisme ou le maquercautage. Il existe un étonnant mêlange de délinquance et de pratiquue littéraire moralement marginale.

23 Claude-François-Xavier Mercier dit de Compiègne (163-1800) est un personnage fascinant qui fut successivement secrétaire de Jaucourt, puis libraire pour vendre ses propres ouvres. Il se spécialisa dans la traduction et la réimpression d'ouvrages licencieux. 
jamais véritablement proposé un système de gouvernement ou une explication du monde, ils illustrent assez bien la montée en puissance de la bohème littéraire, exclue du partage, sans véritable légitimation ni raison d'être. La situation est claire: l'Ancien régime forme des élites et la carrière littéraire apparaît comme un modèle. Et c'est là sans doute un succès public du discours autoglorifiant de la philosophie des Lumières. Car après avoir lu Voltaire, comment ne pas souhaiter vouloir devenir à son tour philosophe? La Philosophie offre un imaginaire de soi et de sa fonction inégalable, semblable peut-être, toutes proportions gardées, à celui que proposaient les prédicateurs des croisades. Il y a là un modèle d'être, une fantasmatique qui triomphe dans ce monde laiicisé. L'écriture devient un métier et une mission. À partir de Rousseau, on va même avancer que quand le métier ne va point, la mission en est d'autant plus certaine. Tout se passe comme si, quel que soit par ailleurs ce qu'ils écrivent, ces hommes se sentaient, conséquence du mépris où on les tient et de leur marginalité, investis d'une part inaliénable de vérité. Voilà une des dimensions peu analysées du rousseauisme littéraire. Ce rousseauisme est celui de cette armée de bohèmes faméliques, mais aussi d'écrivains à succès (absolu ou relatif) comme Rétif de la Bretonne, Louis-Sébastien Mercier et Bernardin de Saint-Pierre.

Leur rousseauisme est revendiqué, proclamé même. Bernardin de Saint Pierre a accompagné Rousseau vieillissant et a donné le récit de ses rencontres avec Jean-Jacques ${ }^{24}$. Louis-Sébastien Mercier est l'auteur d'un Jean-Jacques Rousseau considéré comme l'un des premiers auteurs de la Révolution, où au-delà d'une admiration assez commune, s'exprime son attachement à une façon d'être et de penser la littérature ${ }^{25}$. Quant au rousseauisme de Rétif, il tient moins à son goût de la nature, à son apologie de la vertu, à sa dénonciation du rôle corrupteur de la ville, qu'à sa marginalité d'écrivain-typographe, exclu, en retrait, obsédé par la connaissance de soi et les réminiscences d'une enfance heureuse. On démontrerait facilement que ce qui légitime l'écriture chez Rétif est proprement rousseauiste: la différence, l'altérité, le refus. Ce rousseauisme littéraire demeure encore peu et mal étudié. On

24 Bernardin de Saint-Pierre, "Vieillesse de Jean-Jacques Rousseau ", dans Jean Jacques Rousseau et le pays romand, Genève, 1878.

25 louis-Sébastien Mercier, De Jean-Jacques Rousseau considéré comme l'un des premiers auteurs de la Révolution, Paris, 1791, 2 vol. 
s'est un peu trop exclusivement attaché aux débats politiques sur le Contrat social, aux lectures émues de La nouvelle Héloïse, à la postérité pédagogique ou affective de Rousseau ${ }^{26}$, à la revendication révolutionnaire de sa personne et de ses principes, en oubliant, peu ou prou, que l'écrivain imprime sa marque non seulement dans une écriture du sujet (ce "moi " que revendique Chateaubriand), une sensibilité religieuse au monde et à la nature, une qualité particulière d'émotion, mais aussi dans l'imaginaire même (on aurait peut-être appelé cela en des temps lointains une pratique théorique) de l'écrivain et de l'écriture, posé, consciemment ou non, comme une légitimation, un rapport à la vérité. Ce qui veut dire qu'il faut faire place, au-delà des thématiques nouvelles que la littérature adopte et de la sensibilité des lecteurs à laquelle elle s'adresse, à ce discours réflexif, parfois explicité et souvent implicite de l'écrivain. Une histoire des représentations, ou même de l'idéologie de l'écriture et de ses servants, du rapport privilégié qu'ils entretiennent à la vérité, de leur droit à écrire, à juger et à conseiller, est à faire. Toute une conception particulière d'un savoir spécifique, inaccessible par d'autres voies, se met en place sur la base de ce que le rousseauisme catalyse. Tout écrivain, quelle que soit sa place véritable dans le monde social ou même politique, se donne alors comme autre, marginal, en exil, en rupture, en dissidence, et par là s'autorise à proférer. On aura donc à faire à une mise en scène, parfois grossière, souvent subtile, de ce droit acquis ou conquis ou imposé.

Je me propose ici de brièvement en esquisser la forme chez les héritiers en écriture de Rousseau romancier ou même philosophe, mais aussi là où il semble d'entrée peu présent: chez quelques-uns des journalistes les plus radicaux de la Révolution, Marat et Hébert, rédacteurs comme l'on sait de L'ami du peuple et du Père Duchesne. Pour Marat, j'avancerai quelques hypothèses pour me réserver la possibilité d'une analyse plus précise du Père Ducbesne de Hébert. Dussé-je choquer, la baignoire de Jean-Paul Marat me semble un objet essentiel du rousseauisme de L'ami du peuple. Par elle est rendue possible la rencontre d'une dénonciation et d'une valorisation extrêmes. Elle désigne le malade mais

26 Nous renvoyons aux travaux de Claude Labrosse (cf. note 27) et de JeanJacques 'Tatin, Le contrat social en question: écbos et interprétations du Contrat social de 1766 à la Révolution, Lille, Presses universitaires de Lille, 1989. 
aussi l'exclu, la différence, l'autre épiderme, et en même temps la souffrance, l'écriture quand même, le dévouement malgré tout à la cause militante. La baignoire c'est, en termes différents, la sonde que Rousseau porte sous le manteau d'arménien pour réduire ses difficultés urinaires. Par ailleurs, il est possible de retrouver, terme à terme, dans la démarche mise en cuvre dans L'ami du peuple les transpositions, adaptations et modifications des légitimations rousseauistes. La vertu qui fondait chez Rousseau le rapport privilégié à la vérité est devenue le patriotisme qui confère la vigilance et ordonne le recours incessant à la dénonciation du complot aristocratique. Le patriotisme se démontre par l'appel incessant à détruire les ennemis du peuple, des jacobins et des patriotes et par la vindicte dont Marat est l'objet et qui est sans cesse rappelée. La dénonciation de soi ou de l'autre (émise ou subie) vaut comme preuve de la rectitude de soi. En se disant patriote, en dénonçant comme tels les ennemis du peuple, en démasquant ses faux amis, en subissant les assauts de l'adversaire, on construit sa légitimité. Inscrites dans le champ politique, et la vérité et l'erreur se construisent à partir de nouvelles affirmations. Ce qui sert la volonté et les intérêts du peuple est vrai, le reste relève de la seule erreur. Dans cette perspective la solitude de Marat, son isolement prennent tout leur sens. Elles font de lui une sentinelle, un guetteur avancé, un veilleur attentif dans la nuit de l'Histoire vers qui monte la confiance populaire, dont témoignent les lettres de patriotes, tout aussi vigilants, adressées au rédacteur de L'ami du peuple. Un rapport étroitement politique s'établit entre le publiciste et ses lecteurs qui n'est pas sans rappeler, au-delà de ses traits spécifiques, la relation passionnée qui unit les lecteurs de $L a$ nouvelle Héloüse à Jean-Jacques Rousseau 27.

Le cas de Jacques René Hébert n'est pas strictement identique. Sans doute retrouvera-t-on dans Le père Duchesne ${ }^{28}$ bien des traits relevés dans la stratégie énonciatrice de Marat: dénonciation incessante des ennemis du peuple, droit de vie et de mort attribué au peuple et à son interprète le Père Duchesne, toujours prompt à vous mettre le cou "dans la lunette", relation privilégiée, ainsi qu'en témoignent d'abondantes citations de lettres de

27 Claude Labrosse, Lire au xvir siècle: La nouvelle Héloüse et ses lecteurs, Presses universitaires de Lyon, 1985.

28 Le père Duchesne (1790-1794), introduction d'Albert Soboul, Paris, EDHIS, 10 vol., 1969. 
patriotes, avec le peuple, détenteur de la vérité, d'une extrême vigilance, habile à démasquer le "jean-foutre" aristocrate. On a là des similitudes, dont il faut comprendre qu'elles tiennent d'abord à la nature politique des journaux révolutionnaires, aux rapports de force contemporains, à l'engagement militant de Marat et de Hébert. Mais il convient de différencier les deux positionnements et de définir les mises en œuvre spécifiques, dans un cas et dans l'autre, d'une marginalité énonciative. Hébert a revêtu les oripeaux du Père Duchesne et ce n'est point un hasard. Plus qu'un masque ou un prête-nom, le Père Duchesne est la mise en scène du droit à la parole donné à Hébert. L'homme du peuple, fabricant de poëles, omniscient et omniprésent, peu embarrassé par les nuances, parlant vrai et fort, apparaissant ici comme seul détenteur de la parole mis en scène par le journal révolutionnaire produit l'effet légitimateur de différenciation. La présence de Duchesne, son langage populaire, ici pur effet de style, représentent ce qui, néanmoins, marque la différence avec l'écriture comme artifice et rhétorique. 